\title{
Biomolecule surface patterning may enhance membrane association
}

\author{
Sergey Pogodin, Nigel K. H. Slater ${ }^{\dagger}$ and Vladimir A. Baulin ${ }^{\dagger}$ \\ Departament d'Enginyeria Quimica, Universitat Rovira $i$ Virgili, \\ Av. dels Paisos Catalans 26, 43007 Tarragona, Spain, \\ ${ }^{\dagger}$ Department of Chemical Engineering and Biotechnology, \\ University of Cambridge, Pembroke Street, Cambridge CB2 3RA, \\ UK and ${ }^{\dagger}$ ICREA, Passeig Lluis Companys 23, 08010 Barcelona, Spain *
}

\begin{abstract}
Under dehydration conditions, amphipathic Late Embryogenesis Abundant (LEA) proteins fold spontaneously from a random conformation into $\alpha$-helical structures and this transition is promoted by the presence of membranes. To gain insight into the thermodynamics of membrane association we model the resulting $\alpha$-helical structures as infinite rigid cylinders patterned with hydrophobic and hydrophilic stripes oriented parallel to their axis. Statistical thermodynamic calculations using Single Chain Mean Field (SCMF) theory show that the relative thickness of the stripes controls the free energy of interaction of the $\alpha$-helices with a phospholipid bilayer, as does the bilayer structure and the depth of the equilibrium penetration of the cylinders into the bilayer. The results may suggest the optimal thickness of the stripes to mimic the association of such protein with membranes.
\end{abstract}

Journal link: http://pubs.acs.org/doi/abs/10.1021/nn204736b

Certain biological molecules interact with cell membranes to affect some functional property. Late Embryogenesis Abundant (LEA) proteins are an example of such molecules. These proteins are expressed in plant and some animal cells in response to desiccation conditions and they increase the ability of such cells to withstand desiccation stress $\underline{1}$. They are widespread among higher plants but have also been found in some microorganisms and nematodes exposed to water deficit ${ }^{2}$. LEA proteins may exist in the form of random coils ${ }^{3.4}$ and can spontaneously self-assemble into $\alpha$-helices upon desiccation stress ${ }^{5}$. Such an $\alpha$-helix structure in turn provides an ordered pattern of alternating hydrophilic and hydrophobic stripes that run along the axis of the helix ${ }^{3}$ which may facilitate the interaction and insertion of the protein into the phospholipid membrane. Recently, Popova et al. $\underline{\underline{6}}$ have shown that the fraction of LEA7 protein that takes up the $\alpha$-helix conformation is increased when it is dried in the presence of liposomes, indicating that phospholipid membranes associate closely with LEA proteins and play a critical role in stabilizing their $\alpha$-helix conformation. LEA proteins are amphiphilic and when in the $\alpha$-helix conformation can be approximately described as solid rods with a distinct axial arrangement of hydrophilic and hydrophobic stripes. An entirely distinct class of membrane-active peptides, pore-forming peptides ${ }^{7}$, also have surface patterning with a hydrophilic stripe along the backbone of an $\alpha$-helix. However, the mechanism of association of these proteins with the phospholipid membrane is very different. Membrane pores are formed due to the concerted self-assembly of several peptides on the membrane with a preferential orientation of peptides perpendicular to the bilayer plane; the so-called "Barrel and Stave" mechanism 8 . To allow for such an orientation,

* vladimir.baulin@urv.cat the length of the pore-forming peptides does not usually exceed the thickness of the bilayer ${ }^{9,10}$. In contrast, LEA proteins adopt a more random structure and are much longer ${ }^{11}$. As a consequence they lie as single molecules parallel to the surface of the membrane bilayer. The hydrophobic groups on the side of the backbone control the depth of insertion of the LEA proteins into the bilayer.

To gain insight into the factors that influence the interaction of such a rod with a phospholipid bilayer we have calculated the free energy of association as a function of stripe geometry and position of the rod relative to the center of the bilayer.

In a previous paper ${ }^{12}$ we showed that Single Chain Mean Field (SCMF) theory could be used to calculate the equilibrium structures for insertion of a hydrophobically patterned rod into a phospholipid bilayer with the rod axis oriented perpendicular to the layer. These calculations aimed to determine the effect of patterning upon the puncture of the bilayer by the rod. Here, equilibrium structures of association of a hydrophobically patterned rod with a bilayer are simulated with the rod axis oriented parallel to the layer. These calculations aim to mimic the behaviour of moieties such as LEA proteins that may associate with, but not puncture, bilayers.

\section{RESULTS AND DISCUSSION}

Equilibrium structures of a rigid rod inside a phospholipid bilayer are simulated within the SCMF theory $\underline{13}$. A phospholipid molecule is represented at a coarse-grained level within the three beads model ${ }^{13}$ which is found to describe adequately the mechanical properties of the phospholipid bilayer at equilibrium. The infinite rod lying parallel to the bilayer represents the excluded area for the lipids and water molecules, while the interactions between phospholipids in the bilayer are described through the mean fields. The results of SCMF calculations are presented in Figure 1 and Figure 2, The free energy differences are calculated per unit cylinder length and ne- 


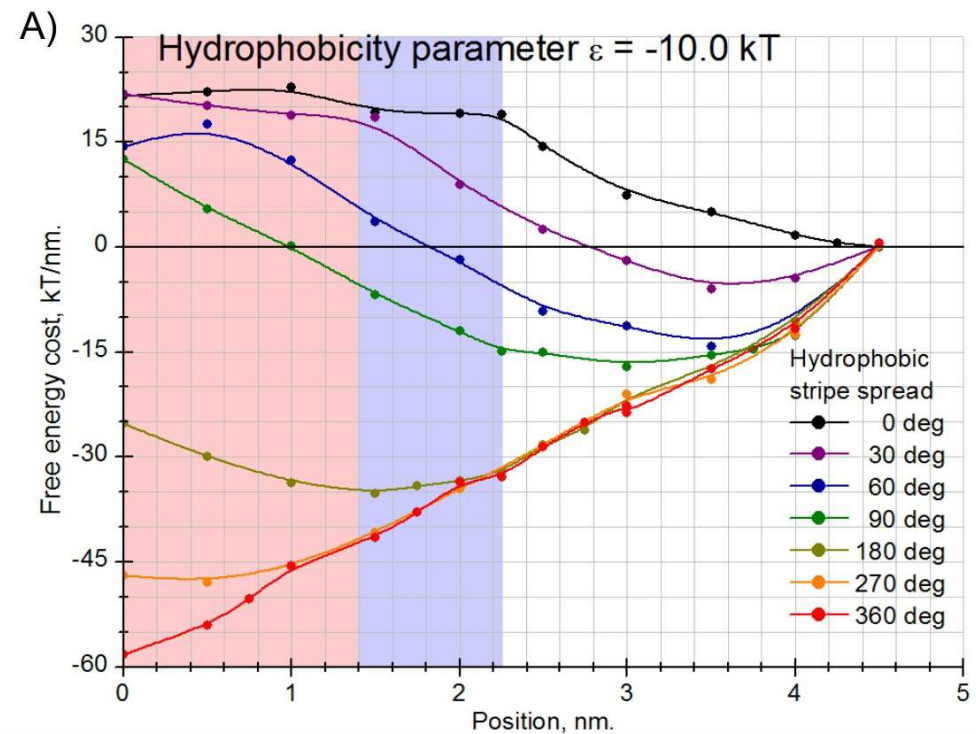

C)

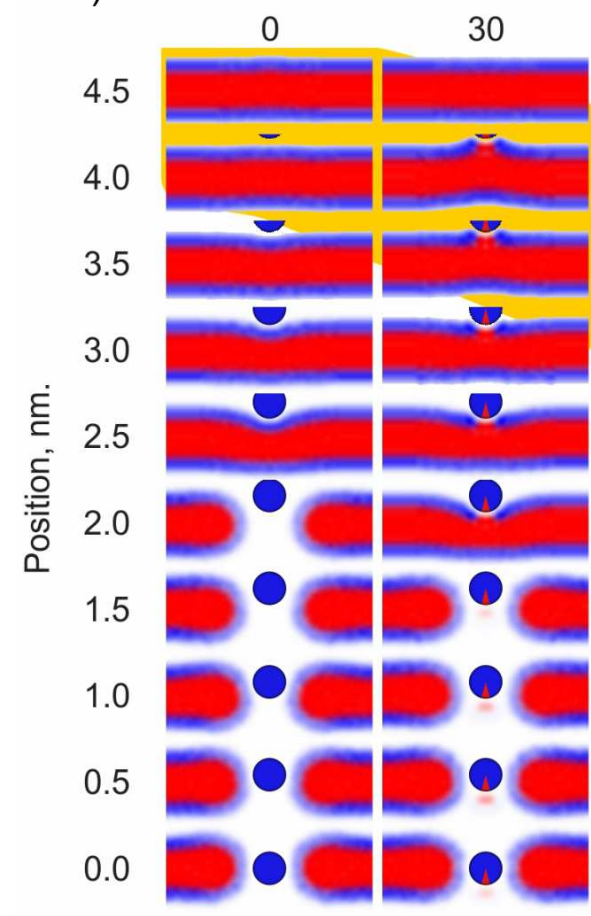

60

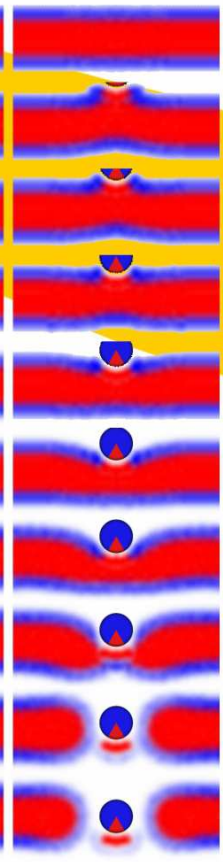

90

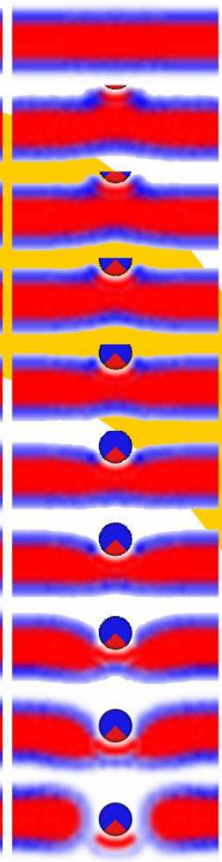

Width of the hydrophobic stripe, deg.

B)
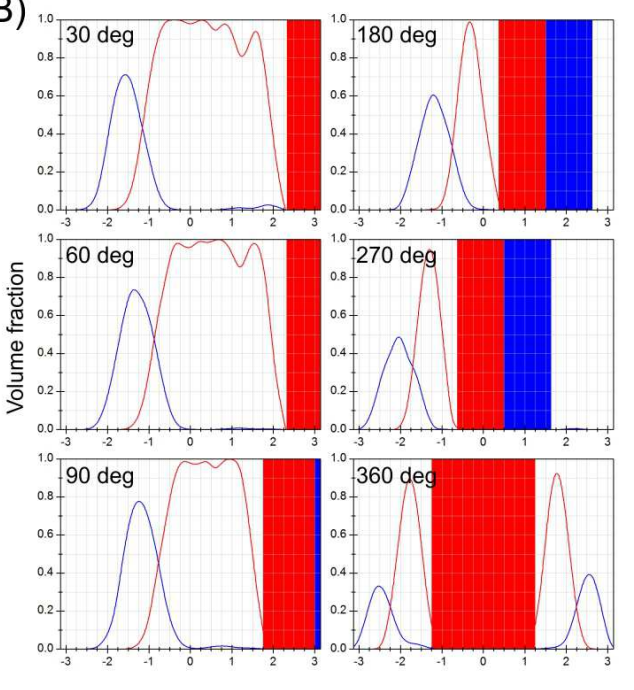

Z-axis, nm.
180

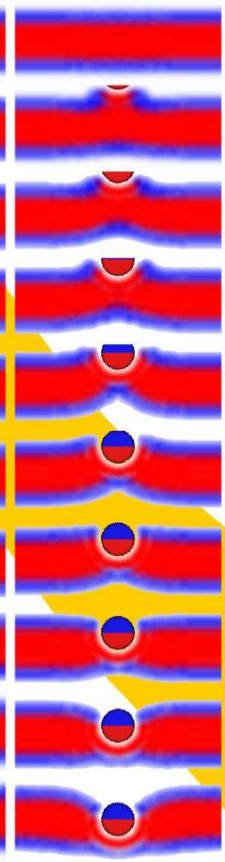

270

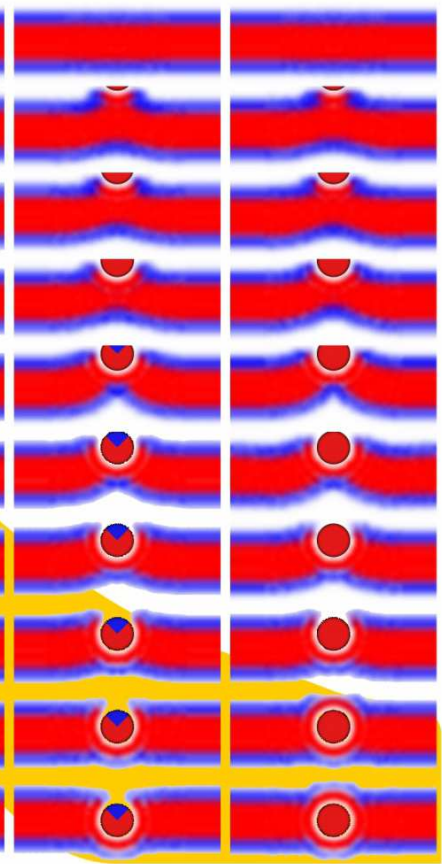

FIG. 1. Insertion of a hydrophilic cylinder, $\varepsilon=0$, with a hydrophobic stripe, $\varepsilon=-10 \mathrm{kT}$, into phospholipid bilayer. (a) The free-energy cost of insertion of cylinders with different width of the hydrophobic stripe (in degrees) as a function of the center position. (b) The concentration profiles of tails (red lines) and heads (blue lines) in the central cross section. Red and blue stripes represent the cylinder with corresponding stripes. (c) Perturbation of the bilayer (blue: heads H, red: tails T) upon insertion of the cylinder. The pink and blue areas at the background show the regions of hydrophobic core and hydrophilic surface of the unperturbed bilayer. Yellow band represents the minimum of the free energy (a), which corresponds to the equilibrium position of the cylinder.

glect end effects. This approximation of infinitely long cylinder is reasonable for cylinders in which the lateral surface area is much larger than the end-cap area. The interaction of lipids with the cylinder are described by a single parameter $\varepsilon$ that is the energy of interaction of phospholipid tails $\mathrm{T}$ with the surface of the cylinder: $\varepsilon=0$ implies only steric repulsion, while negative values reflect the attraction of T-beads to the surface of the cylinder.

Insertion of a completely hydrophilic cylinder, $\varepsilon=0$, into a bilayer is energetically unfavorable (black lines in Figure 1(A) and Figure 2(A)). The energy penalty in- 

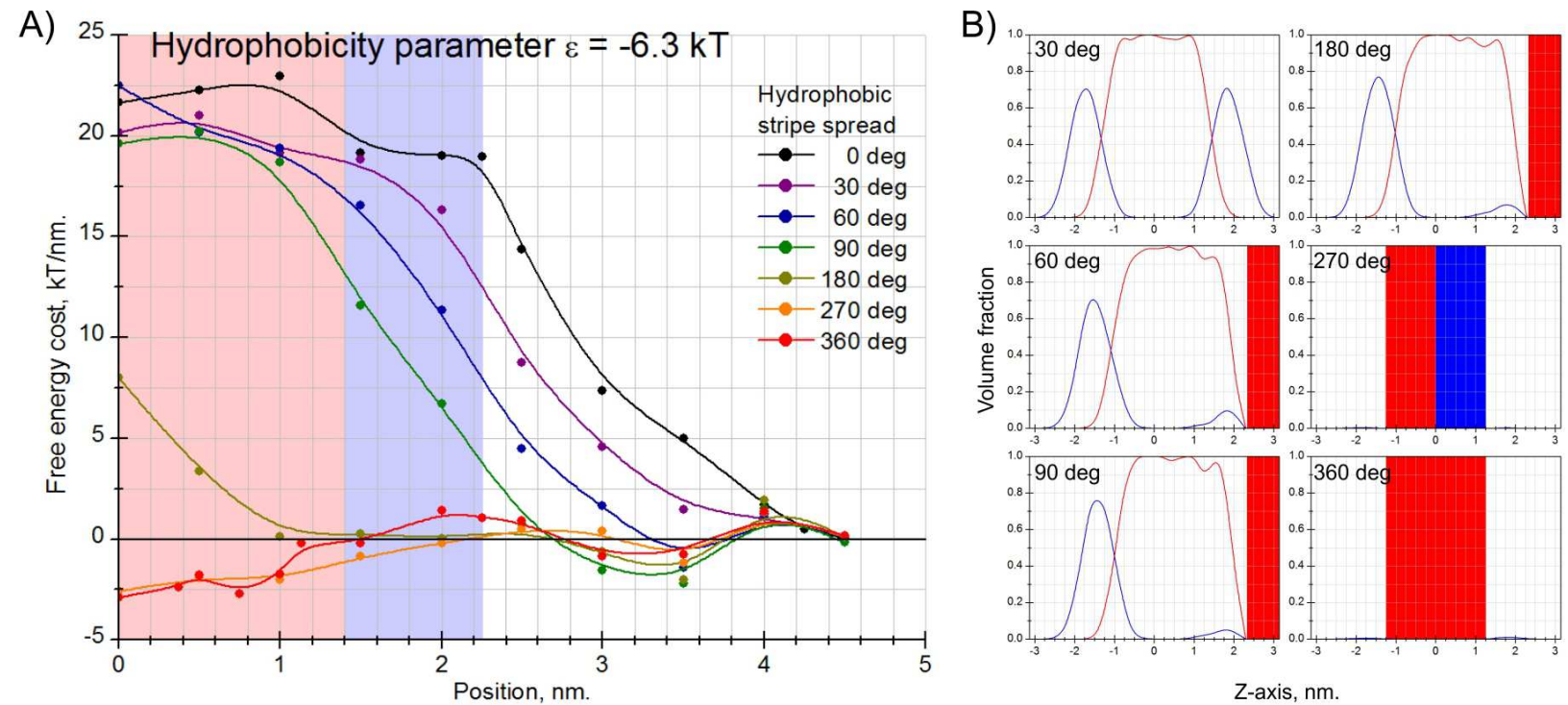

C)

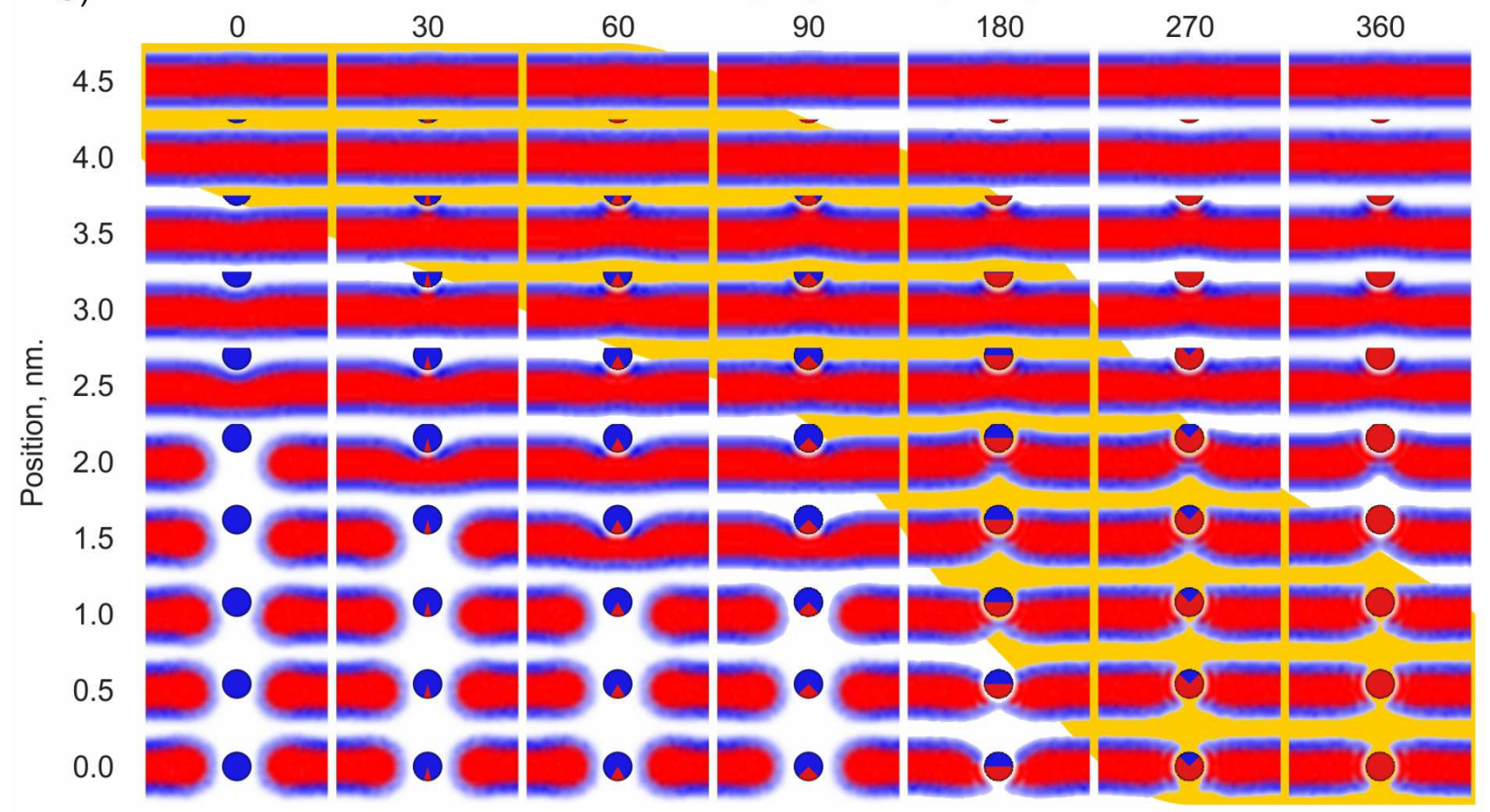

FIG. 2. The same as Figure 1, but the stripe is less hydrophobic, $\varepsilon=-6.3 \mathrm{kT}$.

creases with the length of the cylinder. The hydrophilic cylinder compresses the bilayer at small penetrations and breaks it at distances about $2.0-2.5 \mathrm{~nm}$ from the center. The insertion free energy increases monotonously with depth of penetration and reaches a plateau of height $21.0 \pm 2.0 \mathrm{kT} / \mathrm{nm}$. The rupture of the bilayer is similar to a first order transition in the bulk. Two solutions, one corresponding to the compressed bilayer and another to the broken bilayer, can coexist at some distance, while for other distances one solution is stable and another is metastable. Thus, the cylinder can follow the metastable branch with higher free energy of compressed and intact bilayer before abrupt rupture, accompanied with sudden drop of the free energy. Another reason for the dispersion of the points at the plateau are the error of calculations. Thus, it can be used to estimate the accuracy of the calculations $( \pm 2 \mathrm{kT} / \mathrm{nm})$.

The presence of a parallel hydrophobic stripe on a hydrophilic cylinder makes the penetration of the cylinder inside the bilayer more favorable. This stripe is described by its width or projected angle (in degrees) in Figure 1 and Figure 2. We explore only the orientation with the 


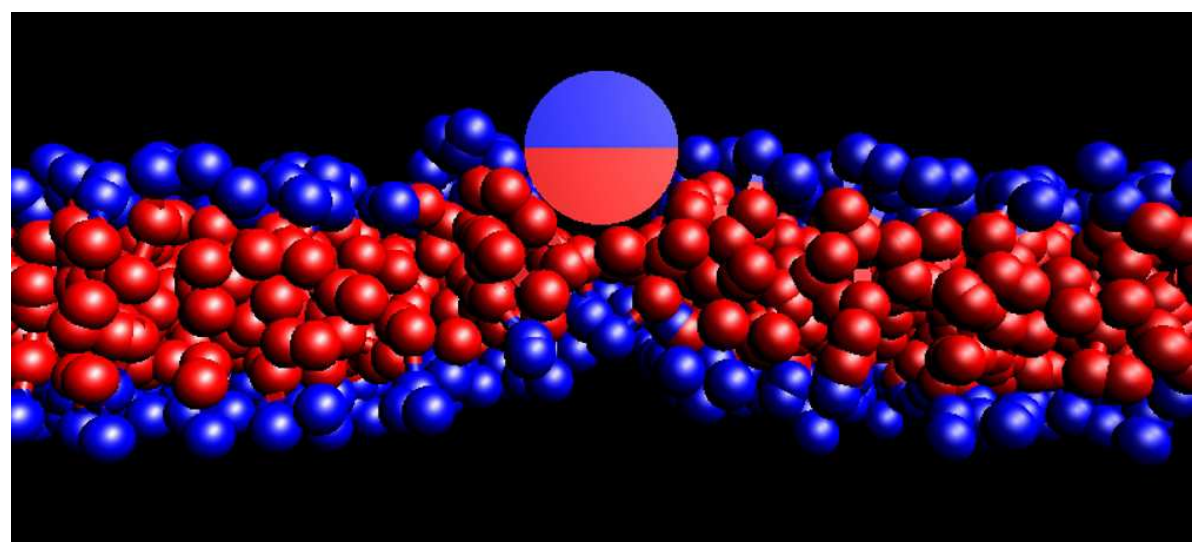

FIG. 3. Amphiphilic cylinder (stripe width 180 degrees, $\varepsilon=-10.0$ ) and the most probable conformations of lipids.

stripe faced down to the bilayer, since this is the lowest energy orientation, but these calculations can also be used to estimate the energy of rotation of the cylinder in the bilayer.

For relatively narrow stripes (with the projected angle in the range $30-90$ degrees) phospholipid heads move apart from the cylinder to accommodate the insertion at low penetrations, which allows for the energetically favorable contact between the stripe and the hydrophobic core of the membrane, and leads to the wetting of the stripe. The contact line moves up the hydrophilic cylinder surface forming a rim and increases the contact with the bilayer core. At higher penetrations the cylinder compresses the bilayer, and, eventually, breaks the membrane. The breakage distance between the cylinder and the membrane centers decreases with an increasing width of the stripe from $1.5-2.0 \mathrm{~nm}$ (the stripe of 30 degrees projected angle) to $0.0-0.5 \mathrm{~nm}$ (90 degrees stripe) and the free energy of penetration becomes lower. The equilibrium position of the cylinder within the bilayer (yellow band in Figure 1(C) corresponding to the minimum of the energy in Figure 1(A)) also shifts to the center of the bilayer with increasing width of the stripe. Thus, the width of the stripe controls not only the hydrophobicity of the cylinder and the insertion energy into the bilayer, but also the equilibrium position with respect to the bilayer core (Figure 1(B)).

The mean field concentration profiles in Figure 1(B) and Figure 1(C) demonstrate that hydrophobic interactions of $\varepsilon=-10.0 \mathrm{kT}$ are high enough for the cylinder to partially adsorb phospholipids from the bilayer. The hydrophobic stripe is covered with the phospholipids even after the rupture of the bilayer. For larger widths of the hydrophobic stripe (180 - 360 degrees), the rupture of both leaflets of the bilayer does not occur and the cylinder is covered by phospholipids in the entire range of penetrations from 0.0 to $4.5 \mathrm{~nm}$ from the bilayer center. The bilayer only bends around the cylinder at small penetrations $1.5-4.0 \mathrm{~nm}$, and covers the whole hydrophobic part of its surface at larger penetrations $0.0-1.5 \mathrm{~nm}$.

The SCMF method provides the probability of con- formations of molecules in a given field. Thus, it allows for visualization of the solutions of the mean field equations in the form of the most probable conformations of molecules in the mean fields that correspond to the solution of the equations 13 . The example of most probable conformations of phospholipids which correspond to the equilibrium position of the amphiphilic cylinder in the bilayer is shown in Figure 3 .

At lower hydrophobicity $(\varepsilon=-6.3 \mathrm{kT}$, Figure 2), the behavior of the system is different in many aspects. The compression of the bilayer for relatively small $(30-90$ degrees) widths of the hydrophobic stripe leads to the rupture of the membrane farther from the bilayer center than for the same stripes with higher hydrophobicity. Similarly, the equilibrium position of the cylinder with the same stripes but lower hydrophobicity is shifted closer to the surface of the bilayer. The overall surface of narrow stripes is too small to allow for the contact with two edges of the broken layer.

Hydrophobic stripes of larger width and $\varepsilon=-6.3 \mathrm{kT}$ induce small perturbations to the structure of the bilayer due to rearrangements of lipids around the stripe at relatively small penetrations. Phospholipid heads pushed apart by the inserted cylinder enable contact of the stripe with the hydrophobic core of the bilayer. Penetration of the cylinder to $2.0-2.5 \mathrm{~nm}$ from the bilayer center, induces the rupture of the bilayer into two parts. Each part of the broken layer contacts with the stripe at their edges. The equilibrium position of the cylinders with large hydrophobic stripes $(180-360$ degrees $)$ is in the center of the bilayer. However, the interaction energy $\varepsilon=-6.3$ is insufficient for phospholipids to cover the cylinder from all sides and the cylinder forms a pore in the bilayer (the central cross-section concentration profiles in Figure 2(B) are different from the corresponding profiles in Figure 1(B) for $180-360$ degrees stripes).

The main difference between $\varepsilon=-10.0 \mathrm{kT}$ and $\varepsilon=$ $-6.3 \mathrm{kT}$ cases is the difference in the free energy of insertion of the cylinder with large widths of the hydrophobic stripe $(180-360$ degrees) (1)(A) and 2(A)). The more hydrophobic cylinder $(\varepsilon=-10.0 \mathrm{kT})$ is fully covered with 


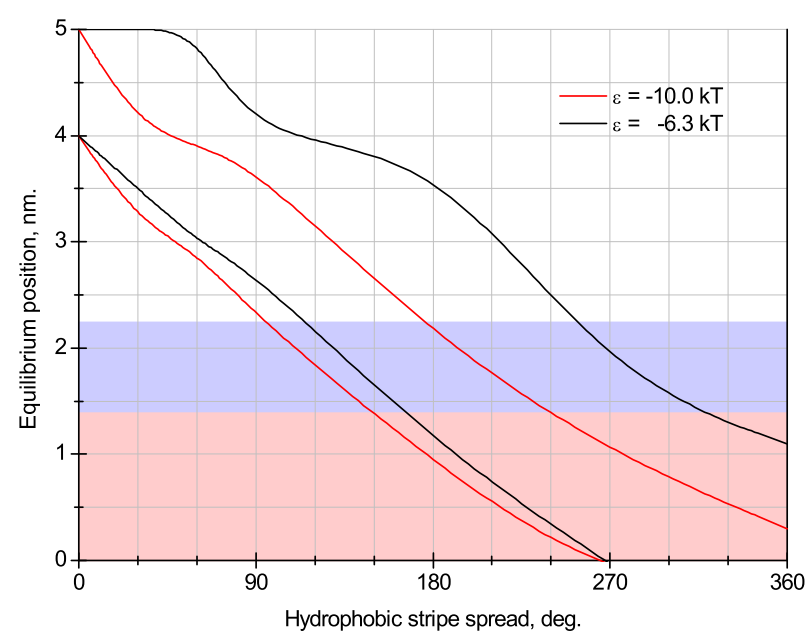

FIG. 4. Range of the cylinder equilibrium position versus width of the hydrophobic stripe on its surface. The equilibrium position is in the region between the two red lines in the case of the stripe with higher hydrophobicity, and between the two black lines in the case of the stripe with lower hydrophobicity. Rose and blue stripes designate the core and the heads regions of the bilayer.

lipids tails and thus its free energy of insertion is much lower than that of the partially covered cylinder with $\varepsilon=-6.3 \mathrm{kT}$. Furthermore, the free energy plots for more hydrophobic cylinder $\varepsilon=-10.0 \mathrm{kT}$ have pronounced minima, while the plots corresponding to $\varepsilon=-6.3 \mathrm{kT}$ have wide regions of relatively constant energies around their minima which correspond to the equilibrium position. Taking into account that the free energy calculations presented here have an accuracy of about \pm 2.0 $\mathrm{kT} / \mathrm{nm}$ and the minima are shallow, one can plot the areas where the free energy cost of the cylinder insertion inside the membrane differs from its minimum value by less than $2.0 \mathrm{kT} / \mathrm{nm}$ (Figure (4). The region of equilibrium positions is wider and is on average shifted from the center of the bilayer for lower hydrophobicity. Thus, our results suggest that the cylinders with wide and low hydrophobicity stripes are able to change their position within a large range around the core of the bilayer with little or no energy cost.

The shallow minima of less hydrophobic $(\varepsilon=-6.3$ $\mathrm{kT})$ and wide $(180-360$ degrees) stripes can also be due to a particularity of the methodology used for the calculations. The vertical size of the simulation box is fixed for both cases and the walls restrict the movement of the bilayer, and so the absence of complete covering of the hydrophobic part of the cylinder with the phospholipids in case of $\varepsilon=-6.3 \mathrm{kT}$ leaves more free space inside the simulation box to bend the bilayer. As a result, small shifts of the cylinder inside the box for less than $1.5 \mathrm{~nm}$ from the equilibrium, lead to bending of the bilayer together with the cylinder without reorganization of the bilayer structure. In contrast, there is almost no free space in the case of the cylinder with $\varepsilon=-10.0 \mathrm{kT}$ and small deviations of the position lead to significant reorganization of lipids covering the hydrophobic stripe, which has a higher free energy cost. However, this difference is only significant for two points of the free energy corresponding to wide stripes.

Although the minimum free energy orientation is with the stripe faced down to the bilayer, an estimate can be made of the free energy of the cylinder rotating around its axis. In the cases of completely hydrophilic, and completely hydrophobic cylinders, there is no changes to the free energy. Rotation of the cylinder with the stripe, leads to contact of the bilayer edges and the hydrophobic core of the cylinder as well as contact between the heads of the bilayer phospholipids with a hydrophilic part of the cylinder. Thus, there is an energy barrier for rotation, which can be estimated as follows. The maximum height of the barrier for rotation of cylinder at position $0.0 \mathrm{~nm}$ from the bilayer center for 90 degrees will be equal, approximately, to the average of the free-energy costs of insertion into this position of completely hydrophobic and completely hydrophilic cylinders (from which we can estimate the energy of rotation for each position). In all others cases the equilibrium positions found for the orientation "stripe-down" will be true equilibrium position for any rotation of the cylinder, because in all these positions the hydrophobic core of the bilayer has the maximum possible contact with the hydrophobic stripe of the cylinder. Hence, any rotation of the cylinder will not increase the contact area, but will significantly deform the bilayer, causing the free energy loss.

\section{CONCLUSION}

Our results demonstrate physical principles of the association of rod-shaped objects with a specific surface pattern and self-assembled phospholipid bilayers. In particular, a hydrophobic stripe along the axis of the cylinder can mimic self-assembled peptides with a secondary periodic structure such as $\alpha$-helices. The position of the cylinder in the bilayer as well as the free energy of association depend on the geometry of the stripe, i.e. the width and the hydrophobicity. This microscopic information, in turn, can be used for design of artificial polymers with enhanced association with phospholipid bilayers.

\section{MATERIALS AND METHODS}

Within the SCMF theory $\stackrel{13}{\underline{13}}$ the phospholipid molecule is modeled at a coarse-grained level, while the interactions between the molecules in the bilayer are represented by the mean fields. The three-beads model ${ }^{12}-15$ describes a phospholipid molecule as a sequence of three spherical beads of a radius $4.05 \AA$, one hydrophilic $(\mathrm{H})$ and two hydrophobic $(\mathrm{T})$, joined consequently with the bond length $10.0 \AA$. Two T-beads of neighboring molecules interact 
with the energy $\varepsilon_{T T}=-2.10 \mathrm{kT}$ if the distance between the centers of the molecules is smaller than $12.15 \AA$. The H-beads interact with implicit solvent molecules with the energy $\varepsilon_{H S}=-0.15 \mathrm{kT}$ if the distance between the centers of the molecules is smaller than $12.15 \AA$. The solvent molecules are considered as spherical beads of the same radius $4.05 \AA$. The phospholipid molecule is free to bend around the central bead.

A cylinder of diameter $24.3 \AA$ and longitudinal hydrophobic stripe on the surface is inserted into phospholipid bilayer at different positions in the simulation box. The position of the cylinder is fixed parallel to the bilayer with the orientation "stripe-down". The hydrophobic part of the surface of the cylinder interacts with the T-beads with the energy $\varepsilon$, if the centers of the molecules are at the distance closer than $8.1 \AA$, while the hydrophilic stripe has only steric repulsion interactions with phospholipids and water molecules. This allows to describe the hydrophobicity of the cylinder with a stripe by its width and a single interaction parameter, $\varepsilon$. We consider an infinite cylinder, where the cylinder length is equal to the size of the simulation box in the corresponding direction, and use the periodic boundary conditions.

In cases the cylinder penetration is unfavorable, the phospholipid bilayer tends to bend and escape the inserted cylinder to minimize the equilibrium free energy. To restrict the bending of the phospholipid bilayer, we introduce hard walls at the top and bottom of the simulation box. This allows to estimate the free energy of insertion at a given position according to procedure described in Ref. 13.

\section{ACKNOWLEDGMENTS}

PS and VAB acknowledge Spanish Ministry of education MICINN project CTQ2008-06469/PPQ. PS, VAB and NKHS thanks the Royal Society for the provision of an International Joint Project grant that facilitated aspects of the work.
[1] Garay-Arroyo, A.; Colmenero-Flores, J. M.; Garciarrubio, A.; Covarrubias, A. A. Highly Hydrophilic Proteins in Prokaryotes and Eukaryotes Are Common during Conditions of Water Deficit. J. Biol. Chem. 2000, 275, 56685674.

[2] Browne, J.; Tunnacliffe, A.; Burnell, A. Anhydrobiosis: Plant Desiccation Gene Found in a Nematode. Nature 2002, 416, 38.

[3] Tolleter, D.; Jaquinod, M.; Mangavel, C.; Passirani, C.; Saulnier, P.; Manon, S.; Teyssier, E.; Payet, N.; Avelange-Macherel, M.-H.; Macherel, D. Structure and Function of a Mitochondrial Late Embryogenesis Abundant Protein Are Revealed by Desiccation. Plant Cell 2007, 19, 1580-1589.

[4] Wise, M. J.; Tunnacliffe, A. POPP the Question: What Do LEA Proteins Do? TRENDS in Plant Science 2004, 9, 13-17.

[5] Goyal, K.; Tisi, L.; Basran, A.; Browne, J.; Burnell, A.; Zurdo, J.; Tunnacliffe, A. Transition from Natively Unfolded to Folded State Induced by Desiccation in an Anhydrobiotic Nematode Protein. J. Biol. Chem. 2003, 278, 12977-12984.

[6] Popova, A. V.; Hundertmark, M.; Seckler, R.; Hincha, D. K. Structural Transitions in the Intrinsically Disordered Plant Dehydration Stress Protein LEA7 Upon Drying are Modulated by the Presence of Membranes. Biochimica et Biophysica Acta 2011, 1808, 1879-1887.

[7] Panchal, R. G.; Smart, M. L.; Bowser, D. N.; Williams, D. A.; Petrou, S. Pore-Forming Proteins and their Application in Biotechnology. Current Pharmac.
Biotechnol. 3, 99-115.

[8] Yang, L.; Harroun, T. A.; Weiss, T. M.; Ding, L.; Huang, H. W. Barrel-Stave Model or Toroidal Model? A Case Study on Melittin Pores. Biophys. J. 2001, 81, 1475-1485.

[9] Illya, G.; Deserno, M. Coarse-Grained Simulation Studies of Peptide-Induced Pore Formation. Biophys. J 2008, 95, 4163-4173.

[10] Gkeka, P.; Sarkisov, L. Interactions of Phospholipid Bilayers with Several Classes of Amphiphilic alpha-Helical Peptides: Insights from Coarse-Grained Molecular Dynamics Simulations. J. Phys. Chem. B 2010, 114, 826839.

[11] Tunnacliffe, A.; Wise, M. J. The Continuing Conundrum Of the LEA Proteins. Naturwissenschaften 2007, 94, 791-812.

[12] Pogodin, S.; Slater, N. K. H.; Baulin, V. A. Surface Patterning of Carbon Nanotubes Can Enhance Their Penetration through a Phospholipid Bilayer. ACS Nano 2011, 5, 1141-1146.

[13] Pogodin, S.; Baulin, V. A. Coarse-Grained Models of Phospholipid Membranes within the Single Chain Mean Field Theory. Soft Matter 2010, 6, 2216-2226.

[14] Pogodin, S.; Baulin, V. A. Can a Carbon Nanotube Pierce through a Phospholipid Bilayer? ACS Nano 2010, 4, 5293-5300.

[15] Pogodin, S.; Baulin, V. A. Equilibrium Insertion of Nanoscale Objects Into Phospholipid Bilayers. CNANO 2011, 7, 721-726. 